\title{
Asymptotic behavior of a Nicholson-Bailey model
}

\author{
Muhammad Naeem Qureshi, Abdul Qadeer Khan* and Qamar Din
}

"Correspondence:

abdulqadeerkhan1@gmail.com

Department of Mathematics,

University of Azad Jammu and

Kashmir, Muzaffarabad, Pakistan
(O2014 Qureshi et al.; licensee Springer. This is an Open Access article distributed under the terms of the Creative Commons Attribution License (http://creativecommons.org/licenses/by/2.0), which permits unrestricted use, distribution, and reproduction in any medium, provided the original work is properly cited.

\begin{abstract}
In this paper, we study the qualitative behavior of a Nicholson-Bailey host-parasitoid model given by

$$
x_{n+1}=R x_{n} e^{-a \sqrt{y_{n}}}, \quad y_{n+1}=x_{n}\left(1-e^{-a \sqrt{y_{n}}}\right),
$$

where $R, a$ and initial conditions $x_{0}, y_{0}$ are positive real numbers. More precisely, we investigate the local asymptotic stability of the unique positive equilibrium point of this system. Some numerical examples are given to verify our theoretical results.

MSC: 39A10; 40A05
\end{abstract}

Keywords: Nicholson-Bailey model; steady-states; local stability

\section{Introduction}

In this paper, we study the dynamics of a Nicholson-Bailey host-parasitoid model which was proposed by Hassel and Varley. In this model the interaction between parasitoids is taken in such a way that the search area per parasitoid is inversely proportional to $\sqrt{y_{n}}$, where $y_{n}$ is the density of parasitoid at year $n$. The model is given by

$$
x_{n+1}=R x_{n} e^{-a \sqrt{y_{n}}}, \quad y_{n+1}=x_{n}\left(1-e^{-a \sqrt{y_{n}}}\right) \text {, }
$$

where $x_{n}$ and $y_{n}$ represent the densities of the host and parasitoid populations at year $n$, respectively, $R$ is the number of offspring of an unparasitized host surviving to the next year. Assuming random encounter between hosts and parasitoids, the probability that a host escapes parasitism can be approximated by $e^{-a \sqrt{y_{n}}}$, where $a$ is a proportionality constant. The probability to become infected is then given by $1-e^{-a \sqrt{y_{n}}}$.

For basic theory of difference equations, we refer to [1-4], and for applications of difference equations, we refer to $[5,6]$. In literature there are many papers on qualitative behavior of biological models [7-18].

Our aim is to investigate the necessary and sufficient condition for local asymptotic stability of unique positive equilibrium point of (1).

\section{Linearized stability}

Let us consider a two-dimensional discrete dynamical system of the form

$$
\begin{aligned}
& x_{n+1}=f\left(x_{n}, y_{n}\right), \\
& y_{n+1}=g\left(x_{n}, y_{n}\right), \quad n=0,1, \ldots,
\end{aligned}
$$


where $f: I \times J \rightarrow I$ and $g: I \times J \rightarrow J$ are continuously differentiable functions and $I, J$ are some intervals of real numbers. Furthermore, a solution $\left\{\left(x_{n}, y_{n}\right)\right\}_{n=0}^{\infty}$ of system (2) is uniquely determined by initial conditions $\left(x_{0}, y_{0}\right) \in I \times J$. An equilibrium point of (2) is a point $(\bar{x}, \bar{y})$ that satisfies

$$
\begin{aligned}
& \bar{x}=f(\bar{x}, \bar{y}), \\
& \bar{y}=g(\bar{x}, \bar{y}) .
\end{aligned}
$$

Definition 1 Let $(\bar{x}, \bar{y})$ be an equilibrium point of system (2).

(i) An equilibrium point $(\bar{x}, \bar{y})$ is said to be stable if for every $\varepsilon>0$ there exists $\delta>0$ such that for every initial condition $\left(x_{0}, y_{0}\right),\left\|\left(x_{0}, y_{0}\right)-(\bar{x}, \bar{y})\right\|<\delta$ implies $\left\|\left(x_{n}, y_{n}\right)-(\bar{x}, \bar{y})\right\|<\varepsilon$ for all $n>0$, where $\|\cdot\|$ is the usual Euclidian norm in $\mathbb{R}^{2}$.

(ii) An equilibrium point $(\bar{x}, \bar{y})$ is said to be unstable if it is not stable.

(iii) An equilibrium point $(\bar{x}, \bar{y})$ is said to be asymptotically stable if there exists $\eta>0$ such that $\left\|\left(x_{0}, y_{0}\right)-(\bar{x}, \bar{y})\right\|<\eta$ and $\left(x_{n}, y_{n}\right) \rightarrow(\bar{x}, \bar{y})$ as $n \rightarrow \infty$.

Definition 2 Let $(\bar{x}, \bar{y})$ be an equilibrium point of a vector map $F(x, y)=(f(x, y), g(x, y))$, where $f$ and $g$ are continuously differentiable functions at $(\bar{x}, \bar{y})$. The linearized system of (2) about the equilibrium point $(\bar{x}, \bar{y})$ is given by

$$
X_{n+1}=F\left(X_{n}\right)=F_{J} X_{n},
$$

where $X_{n}=\left(\begin{array}{c}x_{n} \\ y_{n}\end{array}\right)$ and $F_{J}$ is the Jacobian matrix of system (2) about the equilibrium point $(\bar{x}, \bar{y})$.

Let $(\bar{x}, \bar{y})$ be an equilibrium point of system (1), then one has

$$
\bar{x}=R \bar{x} e^{-a \sqrt{\bar{y}}}, \quad \bar{y}=\bar{x}\left(1-e^{-a \sqrt{\bar{y}}}\right) .
$$

Then it follows that

$$
(\bar{x}, \bar{y})=\left(\frac{R}{R-1}\left(\frac{1}{a} \ln (R)\right)^{2},\left(\frac{1}{a} \ln (R)\right)^{2}\right)
$$

is a unique nontrivial equilibrium point of system (1). Obviously,

$$
(\bar{x}, \bar{y})=\left(\frac{R}{R-1}\left(\frac{1}{a} \ln (R)\right)^{2},\left(\frac{1}{a} \ln (R)\right)^{2}\right)
$$

is a unique positive equilibrium point of system (1) if and only if $R>1$.

The Jacobian matrix of the linearized system of (1) about the fixed point $(\bar{x}, \bar{y})$ is given by

$$
F_{J}(\bar{x}, \bar{y})=\left[\begin{array}{cc}
R e^{-a \sqrt{\bar{y}}} & -\frac{a R \bar{x} e^{-a \sqrt{y}}}{2 \sqrt{\bar{y}}} \\
1-e^{-a \sqrt{\bar{y}}} & \frac{a \bar{x} e^{-a \sqrt{y}}}{2 \sqrt{\bar{y}}}
\end{array}\right] .
$$


Lemma 1 [6] Assume that $X_{n+1}=F\left(X_{n}\right), n=0,1, \ldots$, is a system of difference equations and $\bar{X}$ is the fixed point of $F$. If all eigenvalues of the Jacobian matrix $J_{F}$ about $\bar{X}$ lie inside the open unit disk $|\lambda|<1$, then $\bar{X}$ is locally asymptotically stable. If at least one of them has modulus greater than one, then $\bar{X}$ is unstable.

Lemma $2[4,19]$ Consider the second-degree polynomial equation

$$
\lambda^{2}+p \lambda+q=0
$$

where $p$ and $q$ are real numbers.

(i) A necessary and sufficient condition for both roots of Equation (3) to lie inside the open disk $|\lambda|<1$ is

$$
|p|<1+q<2
$$

In this case the locally asymptotically stable equilibrium $(\bar{x}, \bar{y})$ is also called a sink.

(ii) A necessary and sufficient condition for both roots of Equation (3) to have absolute value greater than one is

$$
|q|>1, \quad|p|<|1+q| .
$$

In this case $(\bar{x}, \bar{y})$ is a repeller.

(iii) A necessary and sufficient condition for one root of Equation (3) to have absolute value greater than one and for the other to have absolute value less than one is

$$
p^{2}-4 q>0, \quad|p|>|1+q| .
$$

In this case the unstable equilibrium $(\bar{x}, \bar{y})$ is called a saddle point.

(iv) A necessary and sufficient condition for a root of Equation (3) to have absolute value equal to one is

$$
|p|=|1+q| .
$$

In this case the equilibrium $(\bar{x}, \bar{y})$ is called a non-hyperbolic point.

\section{Main results}

The following theorem shows the necessary and sufficient condition for local asymptotic stability of the unique positive equilibrium point of (1).

Theorem 1 Assume that $R>1$, then the unique positive equilibrium point

$$
(\bar{x}, \bar{y})=\left(\frac{R}{R-1}\left(\frac{1}{a} \ln (R)\right)^{2},\left(\frac{1}{a} \ln (R)\right)^{2}\right)
$$

of system (1) is locally asymptotically stable if and only if

$$
1<R<R_{0},
$$

where $R_{0}>1$ is the root of the function $F(R):=R \ln (R)-2 R+2$. 
Proof The Jacobian matrix of the linearized system of (1) about the fixed point

$$
(\bar{x}, \bar{y})=\left(\frac{R}{R-1}\left(\frac{1}{a} \ln (R)\right)^{2},\left(\frac{1}{a} \ln (R)\right)^{2}\right)
$$

is given by

$$
F_{J}(\bar{x}, \bar{y})=\left[\begin{array}{cc}
1 & -\frac{R \ln (R)}{2(R-1)} \\
\frac{R-1}{R} & \frac{\ln (R)}{2(R-1)}
\end{array}\right] .
$$

The characteristic polynomial of $F_{J}(\bar{x}, \bar{y})$ about the unique positive equilibrium point

$$
(\bar{x}, \bar{y})=\left(\frac{R}{R-1}\left(\frac{1}{a} \ln (R)\right)^{2},\left(\frac{1}{a} \ln (R)\right)^{2}\right)
$$

is given by

$$
P(\lambda)=\lambda^{2}-\left(1+\frac{\ln (R)}{2(R-1)}\right) \lambda+\frac{R \ln (R)}{2(R-1)} .
$$

Set

$$
p=-\left(1+\frac{\ln (R)}{2(R-1)}\right)
$$

and

$$
q=\frac{R \ln (R)}{2(R-1)} .
$$

Then (5) can be written as

$$
P(\lambda)=\lambda^{2}-p \lambda+q
$$

It follows from Lemma 1 that the unique positive equilibrium point of (1) is locally asymptotically stable if and only if $\left|\lambda_{1}\right|<1$ and $\left|\lambda_{2}\right|<1$, where

$$
\lambda_{1}=\frac{1+\frac{\ln (R)}{2(R-1)}+\sqrt{\left(1+\frac{\ln (R)}{2(R-1)}\right)^{2}-2 \frac{R \ln (R)}{R-1}}}{2}
$$

and

$$
\lambda_{2}=\frac{1+\frac{\ln (R)}{2(R-1)}-\sqrt{\left(1+\frac{\ln (R)}{2(R-1)}\right)^{2}-2 \frac{R \ln (R)}{R-1}}}{2}
$$

are roots of (5). Moreover, $|p|=1+\frac{\ln (R)}{2(R-1)}$ and $1+q=1+\frac{R \ln (R)}{2(R-1)}$. It is easy to see that $|p|<1+q$ for all $R>1$. Take

$$
\Phi(R)=1+\frac{\ln (R)}{2(R-1)}, \quad \Psi(R)=1+\frac{R \ln (R)}{2(R-1)} .
$$




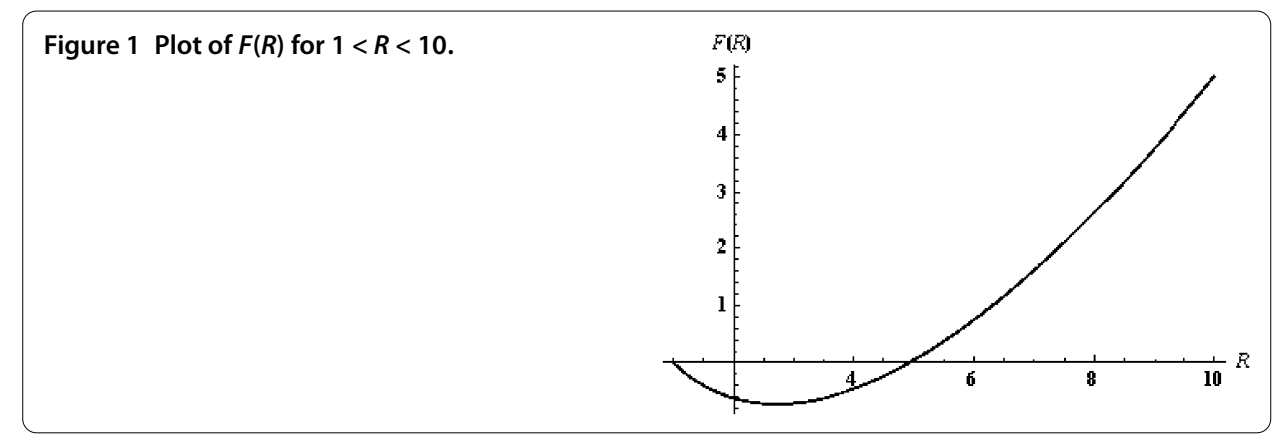

Figure 2 Plots of $\Phi(R)$ and $\Psi(R)$ for $1<R<R_{0}$.

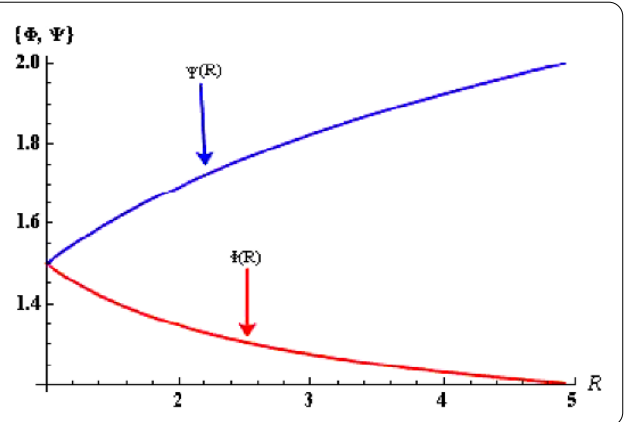

It is enough to show that $\Psi(R)=1+\frac{R \ln (R)}{2(R-1)}<2$, and this holds true if and only if

$$
F(R)=R \ln (R)-2 R+2<0 .
$$

By simple computations one can show that the function $F(R)$ has a unique root in $(1, \infty)$. Furthermore, it follows from Figure 1 and Figure 2 that $F(R)<0$, and equivalently $\Phi(R)<\Psi(R)<2$, if and only if $1<R<R_{0}$, where $R_{0}$ is the unique root of $F(R)$ in $(1, \infty)$, and with mathematica the unique root of function $F(R)$ can be approximated as $R_{0} \approx 4.921553634567504$. Hence, it follows from (i) of Lemma 2 that the unique positive equilibrium point

$$
(\bar{x}, \bar{y})=\left(\frac{R}{R-1}\left(\frac{1}{a} \ln (R)\right)^{2},\left(\frac{1}{a} \ln (R)\right)^{2}\right)
$$

of system (1) is locally asymptotically stable if and only if $1<R<R_{0}$.

Theorem 2 Assume that $R>1$, then the following statements are true.

(i) The unique positive equilibrium point of (1) is a repeller if and only if $R>R_{0}$, where $R_{0}>1$ is the root of the function $F(R)=R \ln (R)-2 R+2$.

(ii) The unique positive equilibrium point of (1) cannot be a saddle point for all $R>1$.

(iii) The unique positive equilibrium point of (1) cannot be non-hyperbolic for all $R>1$.

Proof (i) Assume that $R>1$, then $|q|=\frac{R \ln (R)}{2(R-1)}>1$ if and only if $F(R)=R \ln (R)-2 R+2>0$. 
It follows from Theorem 1 that $F(R)>0$ if and only if $R_{0}<R<\infty$, where $R_{0} \approx$ 4.921553634567504 is the unique root of $F(R)$ in $(1, \infty)$. Moreover,

$$
|p|=1+\frac{\ln (R)}{2(R-1)}<1+\frac{R \ln (R)}{2(R-1)}=|1+q|
$$

for all $R>1$. Hence, from (ii) of Lemma 2 the unique positive equilibrium point of (1) is a repeller if and only if $R>R_{0}$.

(ii) It follows from the fact that

$$
|p|=1+\frac{\ln (R)}{2(R-1)}<1+\frac{R \ln (R)}{2(R-1)}=|1+q|
$$

for all $R>1$. Therefore, the proof of (ii) follows from (iii) of Lemma 2 .

(iii) Obviously, one can prove (iii).

\section{Examples}

In order to verify our theoretical results, we consider some interesting numerical examples in this section. These examples represent different types of qualitative behavior of system (1). First four examples show that the unique positive equilibrium point of system (1) is locally asymptotically stable. Meanwhile, the last two examples show that the positive equilibrium point of system (1) is unstable.

Example 1 Consider system (1) with initial conditions $x_{0}=3.2, y_{0}=2.8$. Moreover, choose the parameters $R=3.99, a=1.5$. Then system (1) can be written as

$$
x_{n+1}=3.99 x_{n} e^{-1.5 \sqrt{y_{n}}}, \quad y_{n+1}=x_{n}\left(1-e^{-1.5 \sqrt{y_{n}}}\right), \quad n=0,1, \ldots,
$$

with initial conditions $x_{0}=3.2, y_{0}=2.8$.

Moreover, in Figure 3 the plot of $x_{n}$ is shown in Figure 3(a), the plot of $y_{n}$ is shown in Figure 3(b) and the phase portrait of system (7) is shown in Figure 3(c).

Example 2 Consider system (1) with initial conditions $x_{0}=1.7, y_{0}=1.5$. Moreover, choose the parameters $R=4.8, a=1.3$. Then system (1) can be written as

$$
x_{n+1}=4.8 x_{n} e^{-1.3 \sqrt{y_{n}}}, \quad y_{n+1}=x_{n}\left(1-e^{-1.3 \sqrt{y_{n}}}\right), \quad n=0,1, \ldots,
$$

with initial conditions $x_{0}=1.7, y_{0}=1.5$.

Moreover, in Figure 4 the plot of $x_{n}$ is shown in Figure 4(a), the plot of $y_{n}$ is shown in Figure 4(b) and the phase portrait of system (8) is shown in Figure 4(c).

Example 3 Consider system (1) with initial conditions $x_{0}=1.9, y_{0}=1.7$. Moreover, choose the parameters $R=4.7, a=1.7$. Then system (1) can be written as

$$
x_{n+1}=4.7 x_{n} e^{-1.7 \sqrt{y_{n}}}, \quad y_{n+1}=x_{n}\left(1-e^{-1.7 \sqrt{y_{n}}}\right), \quad n=0,1, \ldots,
$$

with initial conditions $x_{0}=1.9, y_{0}=1.7$.

Moreover, in Figure 5 the plot of $x_{n}$ is shown in Figure 5(a), the plot of $y_{n}$ is shown in Figure 5(b) and the phase portrait of system (9) is shown in Figure 5(c). 


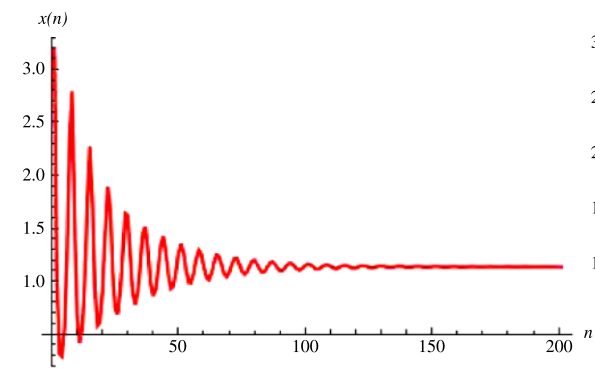

(a) Plot of $x_{n}$ for system (7).

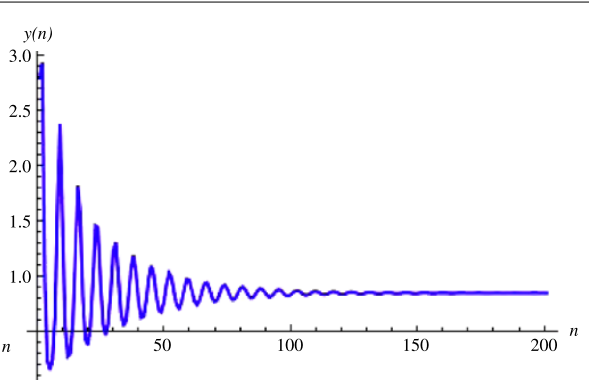

(b) Plot of $y_{n}$ for system (7).

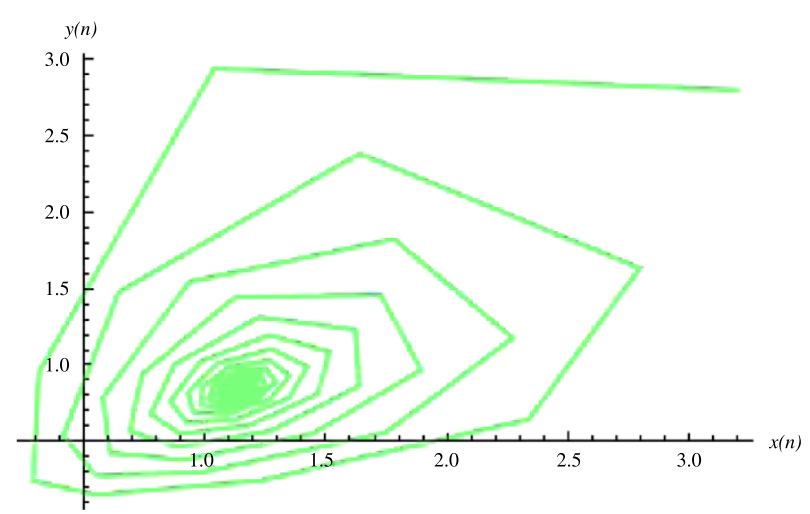

(c) Phase portrait of system (7).

Figure 3 Plots for system (7).

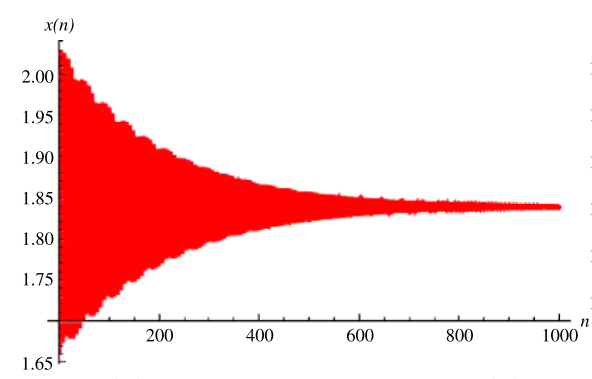

(a) Plot of $x_{n}$ for system (8).

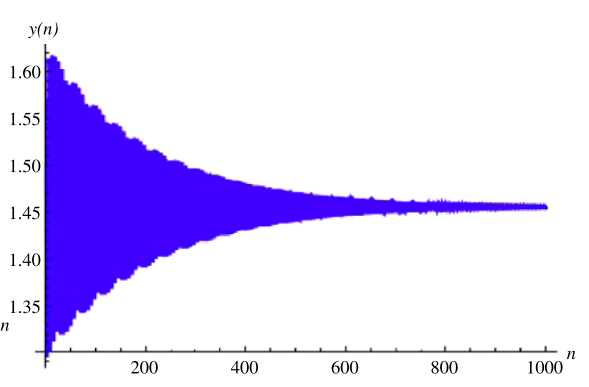

(b) Plot of $y_{n}$ for system (8).

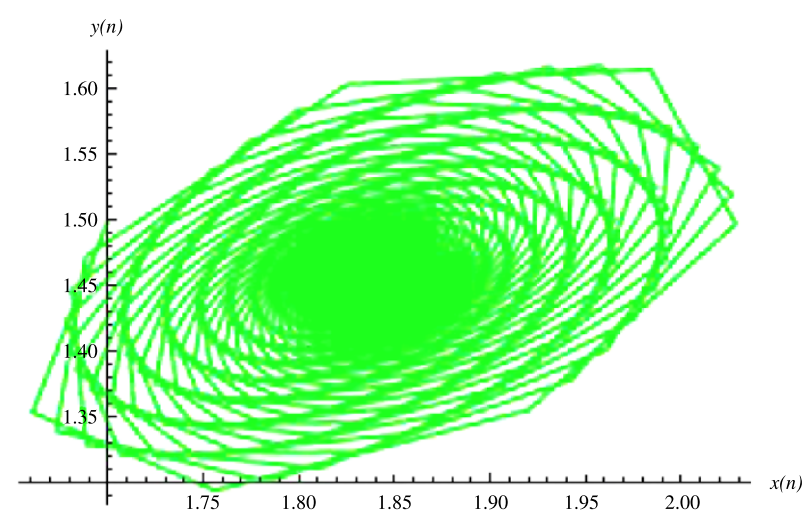

(c) Phase portrait of system (8).

Figure 4 Plots for system (8). 


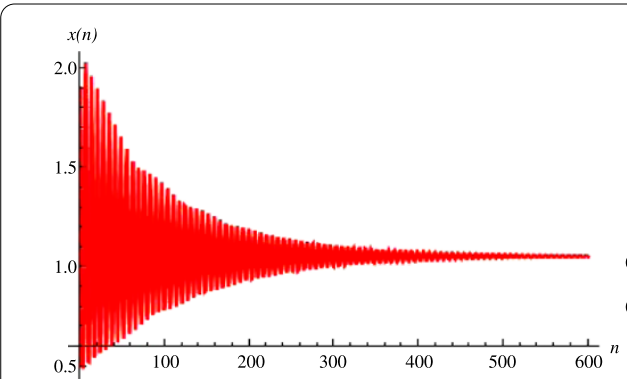

(a) Plot of $x_{n}$ for system (9).

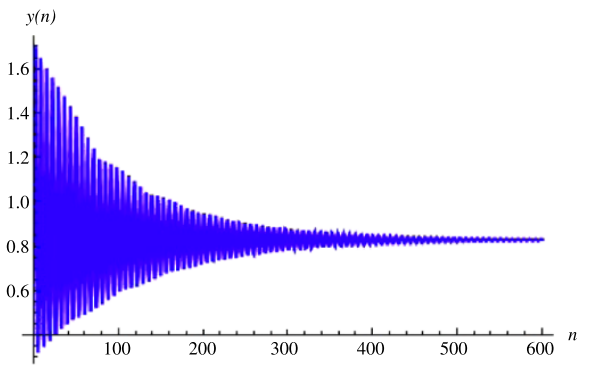

(b) Plot of $y_{n}$ for system (9).

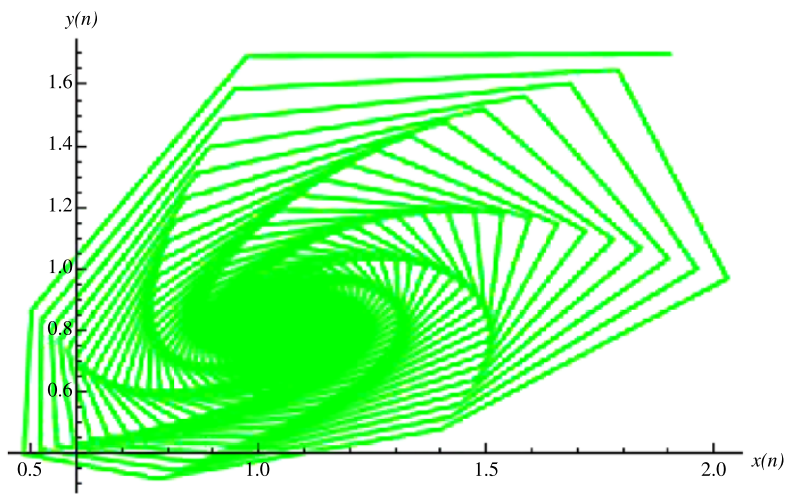

(c) Phase portrait of system (9).

Figure 5 Plots for system (9).

Example 4 Consider system (1) with initial conditions $x_{0}=4.9, y_{0}=2.9$. Moreover, choose the parameters $R=4.2, a=0.8$. Then system (1) can be written as

$$
x_{n+1}=4.2 x_{n} e^{-0.8 \sqrt{y_{n}}}, \quad y_{n+1}=x_{n}\left(1-e^{-0.8 \sqrt{y_{n}}}\right), \quad n=0,1, \ldots
$$

with initial conditions $x_{0}=4.9, y_{0}=2.9$.

Moreover, in Figure 6 the plot of $x_{n}$ is shown in Figure 6(a), the plot of $y_{n}$ is shown in Figure 6(b) and the phase portrait of system (10) is shown in Figure 6(c).

Example 5 Consider system (1) with initial conditions $x_{0}=2.9, y_{0}=4.5$. Moreover, choose the parameters $R=4.99, a=0.5$. Then system (1) can be written as

$$
x_{n+1}=4.99 x_{n} e^{-0.5 \sqrt{y_{n}}}, \quad y_{n+1}=x_{n}\left(1-e^{-0.5 \sqrt{y_{n}}}\right), \quad n=0,1, \ldots
$$

with initial conditions $x_{0}=2.9, y_{0}=4.5$.

Moreover, in Figure 7 the plot of $x_{n}$ is shown in Figure 7(a), the plot of $y_{n}$ is shown in Figure 7(b) and the phase portrait of system (11) is shown in Figure 7(c).

Example 6 Consider system (1) with initial conditions $x_{0}=5.8, y_{0}=6.5$. Moreover, choose the parameters $R=5.2, a=0.7$. Then system (1) can be written as

$$
x_{n+1}=5.2 x_{n} e^{-0.7 \sqrt{y_{n}}}, \quad y_{n+1}=x_{n}\left(1-e^{-0.7 \sqrt{y_{n}}}\right), \quad n=0,1, \ldots
$$




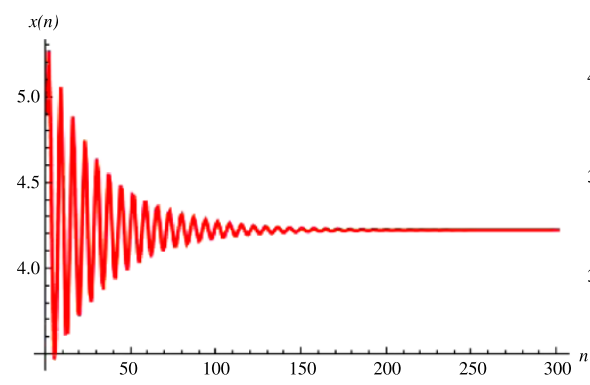

(a) Plot of $x_{n}$ for system (10).

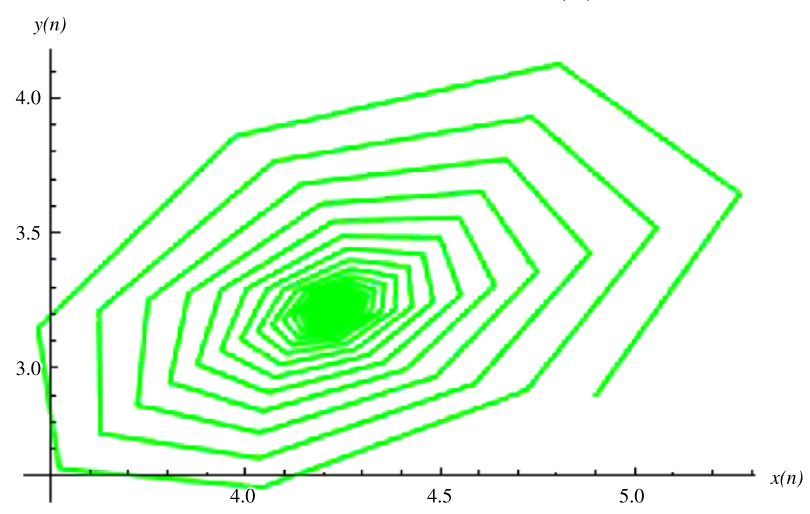

(c) Phase portrait of system (10).

Figure 6 Plots for system (10).

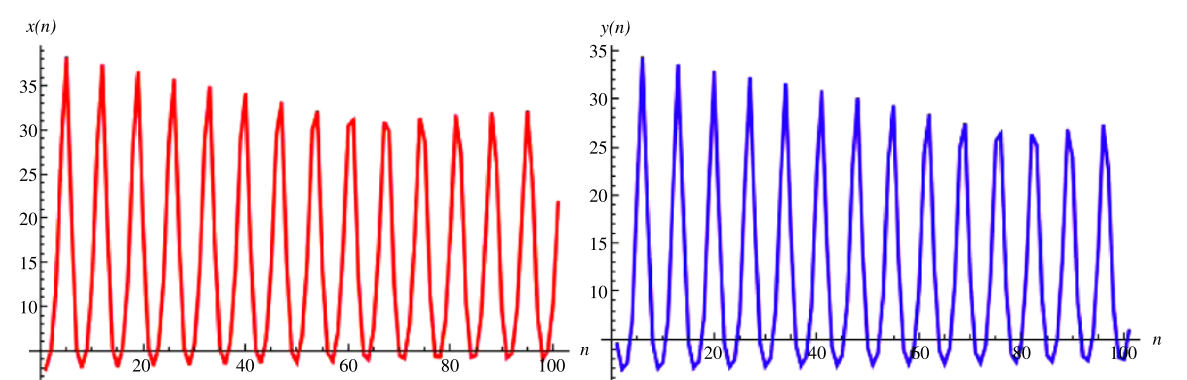

(a) Plot of $x_{n}$ for system (11).

(b) Plot of $y_{n}$ for system (11).

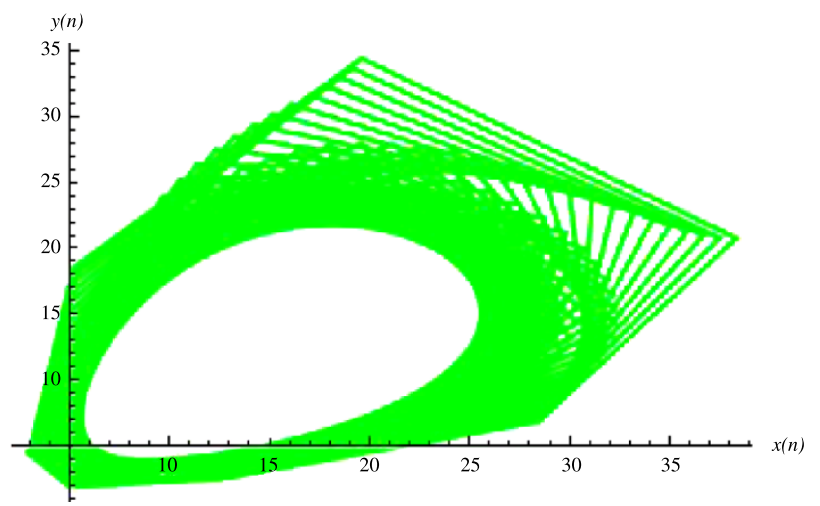

(c) Phase portrait of system (11).

Figure 7 Plots for system (11). 


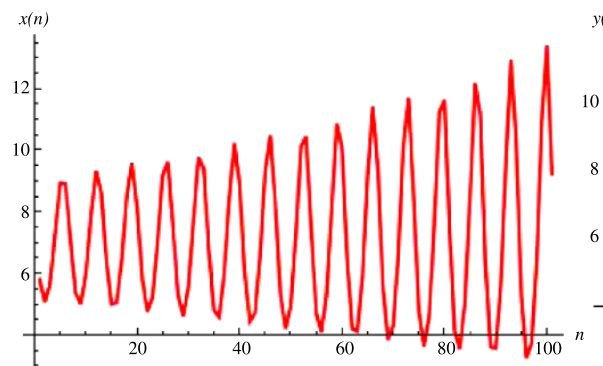

(a) Plot of $x_{n}$ for system (12).

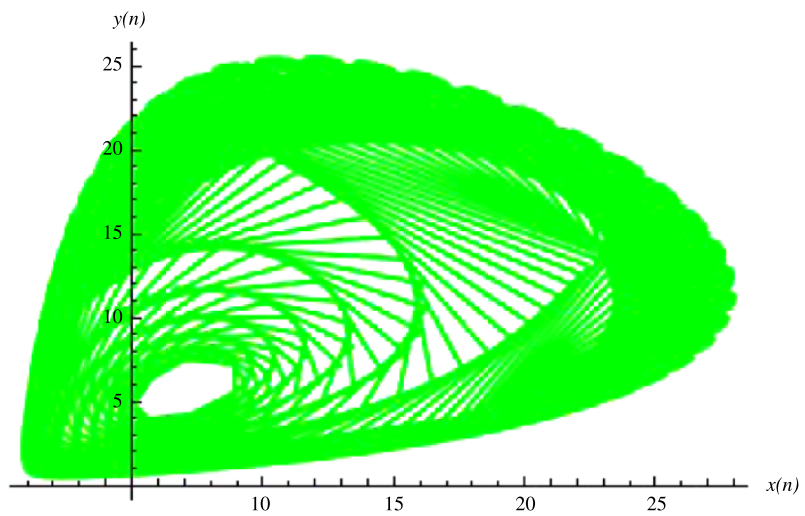

(c) Phase portrait of system (12).

Figure 8 Plots for system (12).

with initial conditions $x_{0}=5.8, y_{0}=6.5$.

Moreover, in Figure 8 the plot of $x_{n}$ is shown in Figure 8(a), the plot of $y_{n}$ is shown in Figure 8(b) and the phase portrait of system (12) is shown in Figure 8(c).

\section{Conclusion and future work}

This work is related to the qualitative behavior of a discrete-time Nicholson-Bailey model. We proved that system (1) has a unique positive equilibrium point which is locally asymptotically stable. The method of linearization is used to prove the local asymptotic stability of the unique equilibrium point. Linear stability analysis shows that the steady states of system (1) are stable under the condition (4) of Theorem 1, i.e., $1<R<R_{0}$, where $R_{0} \approx 4.921553634567504$ is the root of the function $F(R)=R \ln (R)-2 R+2$. Particularly, the condition for local asymptotic stability in population biology is a very interesting mathematical problem. Usually the biologists believe that a unique, positive, locally asymptotically stable equilibrium point in an ecological system is very important in biological point of view. Therefore, it is very important to find conditions which may guarantee the local asymptotic stability of the unique positive equilibrium point of the given system. In the paper, we prove the necessary and sufficient condition for the local asymptotic stability of the unique positive equilibrium point of system (1). Some numerical examples are provided to support our theoretical results. These examples are experimental verifications of theoretical discussions. The Neimark-Sacker bifurcation of system (1) is our next aim to study. 


\section{Competing interests}

The authors have no competing interests.

\section{Authors' contributions}

All authors contributed equally in drafting this manuscript and giving the main proofs

\section{Acknowledgements}

This work was supported by the Higher Education Commission of Pakistan.

Received: 4 October 2013 Accepted: 27 January 2014 Published: 10 Feb 2014

\section{References}

1. Agarwal, RP: Difference Equations and Inequalities: Revised and Expanded, 2nd edn. Dekker, New York (2000)

2. Camouzis, E, Ladas, G: Dynamics of Third-Order Rational Difference Equations with Open Problems and Conjectures. Chapman \& Hall/CRC Press, Boca Raton (2007)

3. Elaydi, S: An Introduction to Difference Equations, 3rd edn. Springer, New York (2005)

4. Grove, EA, Ladas, G: Periodicities in Nonlinear Difference Equations. Chapman \& Hall/CRC Press, Boca Raton (2004)

5. Edelstein-Keshet, L: Mathematical Models in Biology. McGraw-Hill, New York (1988)

6. Sedaghat, H: Nonlinear Difference Equations: Theory with Applications to Social Science Models. Kluwer Academic, Dordrecht (2003)

7. Ahmad, S: On the nonautonomous Lotka-Volterra competition equation. Proc. Am. Math. Soc. 117, $199-204$ (1993)

8. Tang, X, Zou, X: On positive periodic solutions of Lotka-Volterra competition systems with deviating arguments. Proc. Am. Math. Soc. 134, 2967-2974 (2006)

9. Zhou, Z, Zou, X: Stable periodic solutions in a discrete periodic logistic equation. Appl. Math. Lett. 16(2), 165-171 (2003)

10. Liu, X: A note on the existence of periodic solution in discrete predator-prey models. Appl. Math. Model. 34 2477-2483 (2010)

11. Ufuktepe, U, Kapçak, S: Stability analysis of a host parasite model. Adv. Differ. Equ. doi:10.1186/1687-1847-2013-79

12. Din, Q: Dynamics of a discrete Lotka-Volterra model. Adv. Differ. Equ. doi:10.1186/1687-1847-2013-95

13. Din, Q, Donchev, T: Global character of a host-parasite model. Chaos Solitons Fractals 54, 1-7 (2013)

14. Din, Q, Khan, AQ, Qureshi, MN: Qualitative behavior of a host-pathogen model. Adv. Differ. Equ. doi:10.1186/1687-1847-2013-263

15. Din, Q: Global stability of a population model. Chaos Solitons Fractals 59, 119-128 (2014)

16. Allen, LJS, Hannigan, MK, Strauss, MJ: Mathematical analysis of a model for a plant-herbivore system. Bull. Math. Biol. 55(4), 847-864 (1993)

17. Allen, LJS, Hannigan, MK, Strauss, MJ: Development and analysis of mathematical model for a plant-herbivore system. In: Proc. WCNA 92, vol. 4, pp. 3723-3732 (1995)

18. Allen, LJS, Strauss, MJ, Tnorvilson, HG, Lipe, WN: A preliminary mathematical model of the apple twig borer (Coleoptera: Bostricidae) and grapes on the Texas High Planes. Ecol. Model. 58, 369-382 (1991)

19. Kulenović, MRS, Ladas, G: Dynamics of Second Order Rational Difference Equations. Chapman \& Hall/CRC Press, Boca Raton (2002)

10.1186/1687-1847-2014-62

Cite this article as: Qureshi et al.: Asymptotic behavior of a Nicholson-Bailey model. Advances in Difference Equations 2014, 2014:62

\section{Submit your manuscript to a SpringerOpen ${ }^{\ominus}$ journal and benefit from:}

- Convenient online submission

- Rigorous peer review

- Immediate publication on acceptance

- Open access: articles freely available online

- High visibility within the field

- Retaining the copyright to your article 